\title{
Systems medicine for the delivery of better healthcare services - International Federation for Medical and Biological Engineering (IFMBE) perspective
}

\author{
James Goh ${ }^{1}$
}

Received: 30 November 2016 / Accepted: 6 December 2016/Published online: 3 January 2017

(C) IUPESM and Springer-Verlag Berlin Heidelberg 2016

The International Federation on Medical and Biological Engineering was established in 1952, it currently has 60 national and transnational societies. It is the largest federation of its kind in representing and unifying the world-wide Medical and Biological Engineering community. Its mission is to encourage, support the promotion of health and quality of life through advancement of research, development, application and management of technology. True to its goals, it has a global network of professionals at the international level to advance collaboration between national and transnational societies, industry, government and non-governmental organizations engaged in health care and in biomedical research and its applications. IFMBE is a Non-Governmental Organization in official relations with the World Health Organization. In IFMBE, we recognized that the healthcare services landscape is changing rapidly due to multiple factors, ie healthcare economics leading to reformation in the healthcare system, major trends in public health, continuing advances in our understanding of human biology that has the potential impact on medical practice and the development of new innovative technologies for effective and precise diagnosis, treatment and

This article is part of the Topical collection on Systems Medicine

James Goh

biegohj@nus.edu.sg

1 Department of Biomedical Engineering, National University of Singapore, Singapore, Singapore monitoring. As such the field of medical and biological engineering has an important role to constantly attain scientific innovation and translate invention to practice, so as to enhance the healthcare interventions. Even so, there are four key challenges which must be overcome to achieve better healthcare services. The first challenge is comparative effectiveness. Comparative data is necessary and must be available to demonstrate that a new healthcare intervention is an improvement over existing treatment methods. The second challenge is cost containment and reduction. Cost control measures are required to ensure that the public has greater affordable accessibility to high-quality healthcare. The third challenge is global inequities. There is a strong need to bridge the divide between wealthy and poor nations by implementing procedures to support fair healthcare provision. The fourth challenge is accessibility to medicine. It is important to provide adequate access to advanced medical technologies for patients from around the world. In view of these challenges, a systems and multidisciplinary approach would be required to address such complex interactions to achieve better healthcare services.

Compliance with ethical standards

Conflict of interest No conflict of interest.

Funding There is no funding source.

Ethical approval This article does not contain any studies with human participants or animals performed by any of the authors.

Informed consent Not applicable. 